\title{
Angle-Dependent Photoelectron Spectroscopy of Laser-Aligned Atoms: $\mathbf{L i}$
}

\author{
M. Pahler, C. Lorenz, E. v. Raven, J. Rüder, and B. Sonntag \\ II. Institut für Experimentalphysik, Universität Hamburg, 2000 Hamburg 50, Federal Republic of Germany
}

\author{
S. Baier, B. R. Müller, M. Schulze, H. Staiger, and P. Zimmermann \\ Institut für Strahlungs- und Kernphysik, Technishe Universität Berlin, 1000 Berlin 12, Federal Republic of Germany \\ N. M. Kabachnik \\ Institute of Nuclear Physics, Moscow State University, Moscow 119899, Union of Soviet Socialist Republics
}

(Received 9 September 1991)

\begin{abstract}
Combining the linearly polarized high-flux photon beam of a dye laser and the synchrotron radiation of a vacuum-ultraviolet undulator, lithium atoms have been aligned and excited to Li 1 snln'l' core resonances. The angular distribution of the electrons emitted upon the decay of these resonances strongly depends on their symmetry character. Exploiting this dependence, the symmetry of Li 1 snln'l' core resonances has been determined. The results allow for stringent tests of theoretical predictions and demonstrate the great potential of angular-resolved photoelectron spectroscopy on aligned atoms.
\end{abstract}

PACS numbers: $32.80 . \mathrm{Hd}, 32.80 . \mathrm{Dz}, 32.80 . \mathrm{Rm}$

For many years vacuum-ultraviolet (VUV) photoelectron spectroscopy has been established as an extremely powerful method to investigate many-electron effects in atomic photoionization. Energy- and angle-resolved measurements allow for a detailed probing of the dynamics of the ionization, but they still do not provide all the information needed for a full quantum-mechanical characterization of the photoionization process. The necessary additional information has been obtained by detecting the spin polarization of the outgoing electrons [1-3], by measuring the angular distribution of the Auger and photoelectrons $[4,5]$, or by determining the polarization or the angular distribution of the fluorescence radiation $[6,7]$.

Recent progress in laser systems has opened the alternative possibility of preparing the atoms in a well-defined initial state. In a pioneering experiment Meyer et al. [8] succeeded in exciting laser-aligned $\mathrm{Li} 1 s^{2} 2 p^{2} P_{3 / 2}$ atoms to $\mathrm{Li} 1 s n \boldsymbol{n}^{\prime} \boldsymbol{l}^{\prime 2} L_{J}$ core resonances by synchrotron radiation from a bending magnet in an electron storage ring. The limited VUV photon flux only allowed for the detection of the electrons emitted upon the $\mathrm{Li} 1 s n l n^{\prime} l^{\prime 2} L_{J}$ $\rightarrow 1 s^{2}{ }^{\prime} S_{0}+\epsilon l$ decay if the spectrometer integrated over a considerable fraction of $4 \pi$. Using a line source, the first successful attempt to determine the angular distribution of VUV photoelectrons emitted by laser-aligned atoms was made by Kerling, Böwering, and Heinzmann [9]. Because of the limited availability of high-flux polarized VUV line sources, this approach is restricted to a very small number of discrete photon energies. The development of tunable undulator sources has considerably opened the VUV range to the extremely powerful method of angular-resolved photoelectron studies of laser-aligned atoms.

The three-electron lithium atom is the simplest openshell many-electron system and is therefore an excellent model system for a thorough investigation. The great number of narrowly spaced Li $1 s n l n^{\prime} l^{2} L_{J}$ core resonances [10] makes an assignment based on excitation en- ergies alone very difficult, so that an independent check of the symmetry character of the states is of extreme importance for stringent tests of various theories.

The experimental arrangement is schematically depicted in Fig. 1. The lithium atoms emanated from a resistively heated oven. The radiation from a cw dye laser pumped the $\mathrm{Li} 1 s^{2} 2 s^{2} S_{1 / 2} \rightarrow 1 s^{2} 2 p^{2} P_{3 / 2}$ transition at $670.8 \mathrm{~nm}$ with an intensity of $\sim 100 \mathrm{~mW} / \mathrm{mm}^{2}$. Approximately $10 \%$ of the atoms could be prepared in the excited Li $1 s^{2} 2 p^{2} P_{3 / 2}$ state. Transitions from both hyperfine (HF) levels of the ${ }^{2} S_{1 / 2}, F=1,2$ ground state $(\Delta E=820$ $\mathrm{MHz}$ ) were pumped simultaneously. By measuring the degree of polarization or the angular distribution of the resonant fluorescence radiation, the alignment of the excited state was determined. The value of -0.7 achieved for the alignment parameter $\mathcal{A}_{20}$ [11] agrees with the value estimated for a $J=\frac{3}{2}$ resonance, taking the

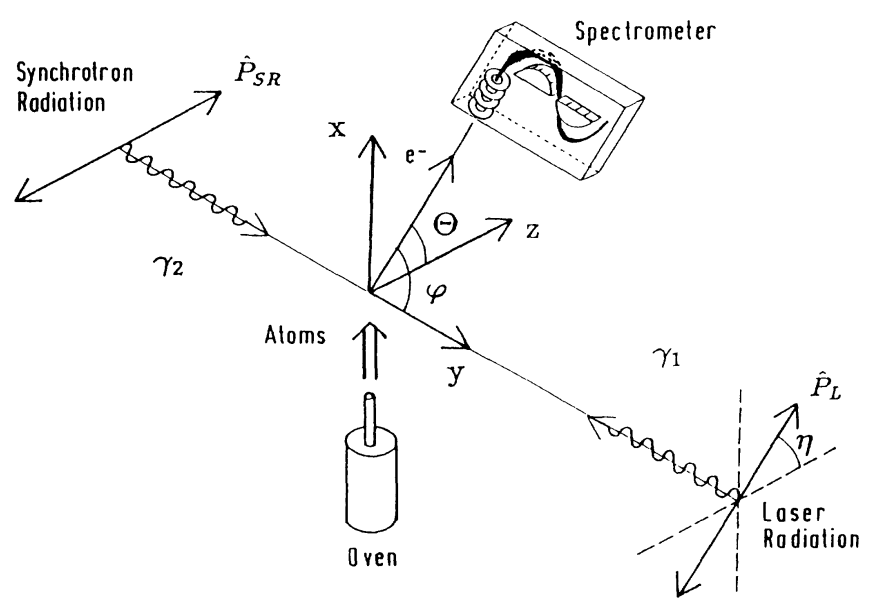

FIG. 1. Scheme of the experimental arrangement. The synchrotron radiation and the laser radiation propagate in opposite directions along the $Y$ axis. Both photon beams intersect the beam of $\mathrm{Li}$ atoms in the source volume of a pseudohemispherical electron analyzer [13] which can be rotated around the $Y$ axis. 
coherent excitation of the HF levels into account [12]. From this aligned ${ }^{2} P_{3 / 2}$ state the $\mathrm{Li} 1 s n l n^{\prime} l^{\prime}{ }^{2} S_{1 / 2},{ }^{2} D_{3 / 2,5 / 2}$ core resonances were excited using the high-flux undulator radiation at BESSY with a bandpass of $0.3 \mathrm{eV}$ at $\hbar \omega=60 \mathrm{eV}$ and a linear polarization of $98 \%$. The electrons were analyzed by a simulated hemispherical spectrometer [13] (angular acceptance $\pm 3^{\circ}$ ) with a fourelement electrostatic entrance lens. Operating the analyzer in a nonretarding mode, an energy resolution of $2 \%$ was achieved. This spectrometer could be rotated around the photon beams in a plane perpendicular to the light beams. In this plane $\theta$ measured the angle between the outgoing electrons and the polarization axis of the undulator radiation. The angle between the two polarization axes was given by $\eta$. The system was operated in two modes: (i) setting the spectrometer at a fixed angle $\Theta$ and recording the electron intensity as a function of $\eta$ by rotating the polarization axis of the laser, and (ii) keeping $\eta$ fixed and registering the electron intensity as a function of $\Theta$ by rotating the electron analyzer.
In the $L S$ coupling approximation the probability for the emission of an electron in the direction $\mathbf{n}_{\ell}$ is given by

$$
W\left(\mathbf{n}_{e}\right)=\sum_{K_{L}} \sqrt{2 \pi} \alpha_{K_{L}} \sum_{Q_{L}} \rho_{K_{L} Q_{L}} \mathcal{Y}_{K_{L} Q_{L}}\left(\mathbf{n}_{e}\right)
$$

where $\alpha_{K_{l}}$ is a kinematic parameter including the square of the Auger matrix elements $\left|M_{l}\right|^{2}, \rho_{K_{L}} Q_{L}$ are the statistical tensor components of the decaying states [14], and $\mathcal{Y}_{K_{L}, Q_{L}}$ are the spherical harmonics. The tensor rank $K_{L}$. is restricted to $0 \leq K_{L} \leq 2 L$ and is even. $L$ is the total orbital angular momentum of the decaying state. In the case of the Li $1 s n l n^{\prime} l^{\prime}$ core resonances the complexity is greatly reduced. There is only the decay of the ${ }^{2} S_{1 / 2}$ and ${ }^{2} D_{3 / 2,5 / 2}$ states into the ${ }^{\prime} S_{0}$ state of the $\mathrm{Li}^{+} 1 s^{2}$ ion. The autoionization of the ${ }^{2} P_{1 / 2,3 / 2}$ resonances is forbidden due to parity and angular momentum selection rules. The angular distribution of the outgoing electrons in the $X-Z$ plane is given by, for (a) ${ }^{2} S_{1 / 2} \rightarrow{ }^{1} S_{0}+\epsilon l$,

$$
W=\sqrt{2}\left|M_{0}\right|^{2} \rho_{00} \bar{P}_{00},
$$

and for (b) ${ }^{2} D_{3 / 2,5 / 2} \rightarrow{ }^{1} S_{0}+\epsilon l$,

$$
\begin{aligned}
W(\Theta)= & \sqrt{2}\left|M_{2}\right|^{2} \rho_{00} \bar{P}_{00}-(2 / \sqrt{7})\left|M_{2}\right|^{2}\left[\rho_{20} \bar{P}_{20}(\cos \Theta)+2 \rho_{21} \bar{P}_{21}(\cos \Theta)+2 \rho_{22} \bar{P}_{22}(\cos \Theta)\right] \\
& +(2 / \sqrt{7})\left|M_{2}\right|^{2}\left[\rho_{40} \bar{P}_{40}(\cos \Theta)+2 \rho_{41} \bar{P}_{41}(\cos \Theta)+2 \rho_{42} \bar{P}_{42}(\cos \Theta)\right]
\end{aligned}
$$

with $\bar{P}_{K_{L} Q_{L}}$ the normalized associated Legendre functions. The dependence on $\eta$ is described by the statistical tensor components $\rho_{K_{L}} Q_{L}$.

The spectrum of electrons emitted upon the $\mathrm{Li}$ $1 s^{2} 2 s^{2} S_{1 / 2} \rightarrow 1 s^{2} 2 p^{2} P_{1 / 2} \rightarrow 1$ snln $l^{\prime}{ }^{2} S_{1 / 2},{ }^{2} D_{3 / 2,5 / 2} \rightarrow 1 s^{2}-$ ' $S_{0}+\epsilon l$ excitation and autoionization sequence in the energy range $E=60.35-66.35 \mathrm{eV}$ has been studied by Meyer et al. [8]. This spectrum comprises a series of discrete autoionization resonances. The $\mathrm{Li} 1 s 2 p^{22} D_{3 / 2,5 / 2}$ resonance at $E=61.06 \mathrm{eV}$ is by far the strongest and is energetically well separated from the other resonances. There is general agreement on the assignment of this resonance [10,15-17]. In order to test our approach we started with the investigation of the angular distribution of the electrons emitted upon the $\mathrm{Li} 1 s 2 p^{22} D_{3 / 2,5 / 2} \rightarrow \mathrm{Li}$ $1 s^{2}{ }^{\prime} S_{0}+\epsilon d$ autoionization. The intensity of the outgoing electrons as a function of the spectrometer angle $\Theta$ for three different orientations of the two polarization axes $\left(\eta=0^{\circ}, 45^{\circ}, 90^{\circ}\right)$ is shown in Fig. 2. Between the angles $\Theta=80^{\circ}$ and $115^{\circ}$ the spectrometer was blocked by the cold trap; thus in this range no data could be taken. The bottom spectrum in Fig. 2 was recorded at an angle $\eta=90^{\circ}$. Changing the angle $\eta$ to $45^{\circ}$ and $0^{\circ}$, the spectra shown at the center and at the top of Fig. 2 have been obtained. The solid lines in Fig. 2 represent least-squares fits of the experimental data by the theoretical angular distribution $W(\Theta)$ given by formula (3). These results already prove the $D$ character of the resonance, because the angular distribution of the outgoing electrons for an $S$ resonance should not depend on the angle $\Theta$. Another possibility to check the assignment of the resonance is to measure the electron intensity as a function of the angle $\eta$

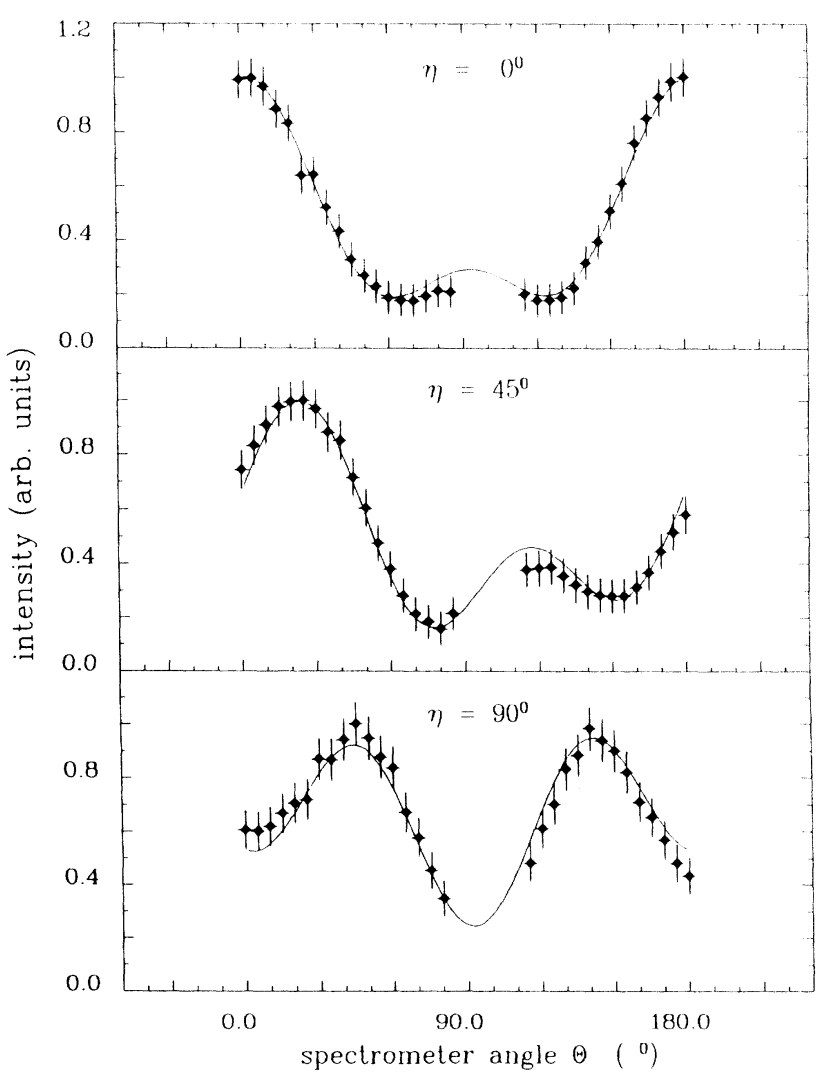

FIG. 2. Intensity of the electrons emitted upon the process $1 s^{2} 2 p^{2} P_{3 / 2} \rightarrow 1 s 2 p^{2}{ }^{2} D_{3 / 2.5 / 2} \rightarrow 1 s^{2} S_{0}+\epsilon l$ vs the spectrometer angle $\Theta$ for three different angles between the two polarization axes $\left(\eta=0^{\circ}, 45^{\circ}, 90^{\circ}\right)$. The solid lines represent a fit of the data by formula (3). 
between the polarization axes of the two radiation fields. The data obtained in different measurements, varying $\eta$ or $\boldsymbol{\theta}$ within the experimental uncertainties, can be well approximated by the theoretical distribution $W\left(\mathbf{n}_{e}\right)$ for a $D$ resonance.

Confident in our approach we turn to the resonance at $E=63.54 \mathrm{eV}$, which is well suited for demonstrating the potential of the angular-resolved photoelectron spectroscopy of laser-aligned atoms. Measurements of the $\Theta$ dependence show an intensity variation of the outgoing electrons which is definitely larger than expected for an $S$ resonance but at the same time significantly smaller than for a $D$ autoionization resonance. Also the $\eta$ dependence of the electron intensity cannot be reconciled with the decay of a pure $S$ or $D$ resonance. The upper spectrum in Fig. 3 shows the electron intensity as a function of $\eta$ measured for a spectrometer angle $\Theta=45^{\circ}$. The experimental results can be explained by assuming that the decay of two closely spaced $S$ and $D$ resonances gives rise to the electron emission. The existence of an $S$ and a $D$ resonance in this energy range is supported by several experimental $[10,18-21]$ and theoretical [10,15,16,21] results, though the assignments of the resonances differ. Based on the calculations of Weiss (see Ref. [10]), Wakid, Bhatia, and Temkin [15], and Chung [16] we expect the transitions $\mathrm{Li} \quad 1 s^{2} 2 p^{2} P_{3 / 2} \rightarrow 1 s 2 p^{2} S_{1 / 2}$; $1 s 2 s\left({ }^{3} S\right) 4 d^{2} D_{3 / 2,5 / 2}$ to contribute. As a result of the bandwidth of $0.3 \mathrm{eV}$ of the VUV radiation both resonances are excited in our experiment.

By superimposing the angular distributions of the electrons emitted upon the decay of an $S$ and a $D$ resonance, reasonable agreement with experimental results can be achieved. The two distributions and their sum are given in Fig. 3. The relative weight of the two distributions is determined by the $\mathrm{Li} 1 s^{2} 2 p^{2} P_{3 / 2} \rightarrow 1 s 2 p^{22} S_{1 / 2}$; $1 s 2 s\left({ }^{3} S\right) 4 d^{2} D_{3 / 2,5 / 2}$ excitation probabilities. Based on the assumption that the resonances coincide, the ratio of the dipole matrix elements $\left|\left\langle\gamma P\left\|r^{(1)}\right\| \gamma^{\prime} S\right\rangle\right|^{2}$ and $\left|\left\langle\gamma P \| r^{(i)}|| \gamma^{\prime} D\right\rangle\right|^{2}$ is close to 1 . This result is also supported by our Hartree-Fock calculations. A comparison of the experimental resonance profile with the superposition of two Gaussians of the deduced intensity ratio and a half-width of $0.16 \mathrm{eV}$ shows no marked deviation if the two Gaussians are separated by less than $0.1 \mathrm{eV}$.

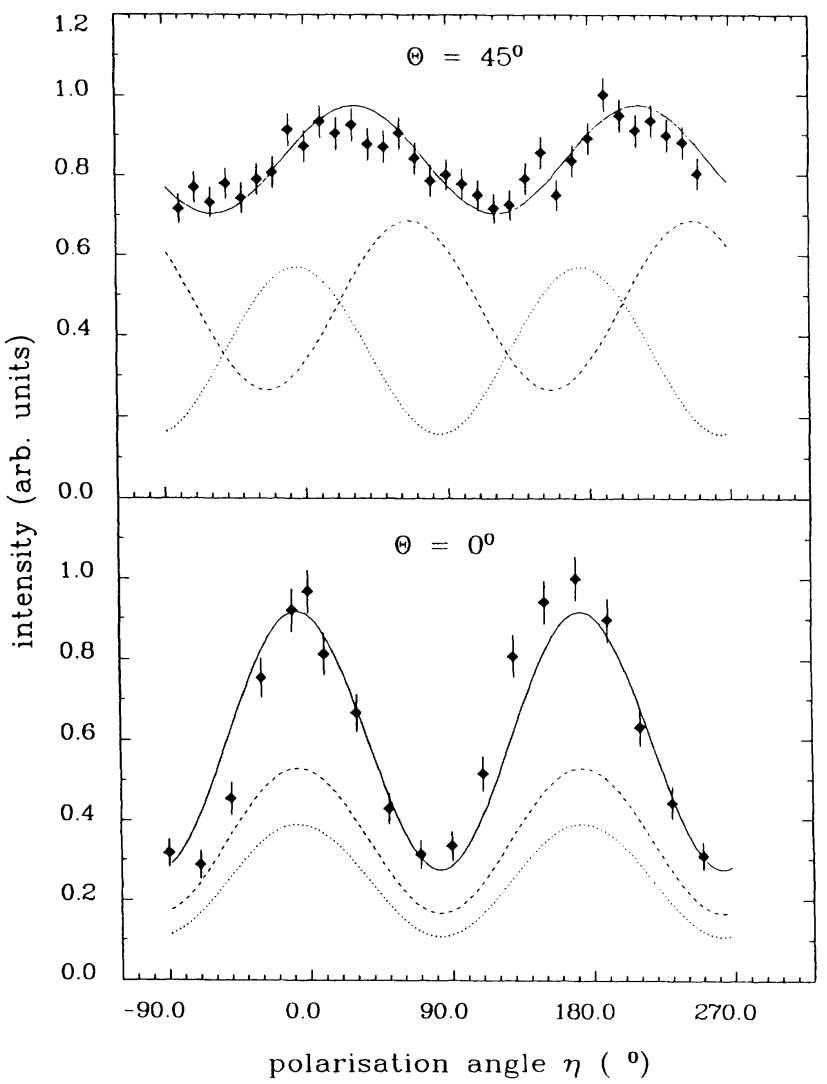

FIG. 3. Intensity of the electrons emitted upon the decay of the resonance at $E=63.54 \mathrm{eV}$ as a function of the angle $\eta$ between the polarization axes of the two radiation fields. The solid line represents the superposition of the angular distributions calculated for the decay of an $S$ [formula (2)] and a $D$ resonance [formula (3)]. The two distributions are given separately by the dotted ( $S$ resonance) and dashed ( $D$ resonance) curves. Upper spectrum: Spectrometer angle $\Theta=45^{\circ}$. Lower spectrum: $\Theta=0^{\circ}$.

TABLE I. Li $\mid s n l{ }^{\prime} l^{\prime}$ resonance energies and assignments determined in this work. For comparison the results of several theoretical studies are included [10,15-17]. The energies are relative to the $1 s^{2} 2 s^{2} S_{1 / 2}$ ground state. The numbers in parentheses give the uncertainty of the last digit.

Resonance energy from ground state (eV)

\begin{tabular}{lcccccc}
\hline \hline & \multicolumn{5}{c}{ Resonance energy from ground state (eV) } \\
\multicolumn{1}{c}{ Assignment } & {$[10]$} & {$[16]$} & {$[17]$} & {$[15]$} & Assignment & Energy (eV) \\
\hline $1 s 2 p^{2}{ }^{2} D$ & 61.11 & 61.071 & 61.177 & 61.064 & ${ }^{2} D$ & $61.06(1)$ \\
$1 s 2 s\left({ }^{3} S\right) 3 d^{2} D$ & 62.98 & 62.909 & 63.008 & 62.914 & ${ }^{2} D$ & $62.90(2)$ \\
$1 s 2 s\left({ }^{1} S\right) 3 s^{2} S$ & 63.23 & $\ldots$ & 63.425 & 63.159 & ${ }^{2} S$ & $63.11(2)$ \\
$1 s 2 p^{2} S$ & 63.50 & $\ldots$ & $\ldots$ & 63.485 & ${ }^{2} S+{ }^{2} D$ & $63.54(2)$ \\
$1 s 2 s\left({ }^{3} S\right) 4 d^{2} D$ & 63.62 & 63.567 & $\ldots$ & 63.676 & ${ }^{2} D$ & $65.60(2)$ \\
$1 s 2 p\left({ }^{2} P\right) 3 p^{2} D$ & $\cdots$ & 65.654 & $\cdots$ & 65.625 & ${ }^{2} D$ & \\
\hline \hline
\end{tabular}


Table I summarizes our results for the most prominent resonances. For comparison the assignment and the energies obtained by several calculations are included. Our results confirm most of the assignments. Even for the resonance at $63.54 \mathrm{eV}$ the theoretical predictions are consistent with the interpretation of the experimental results discussed above.

Angular-resolved VUV photoelectron spectroscopy of laser-aligned free atoms is a new and very promising method for the study of core excitations. Our results demonstrate the feasibility of the method and provide a foretaste for the wealth of information accessible with the new generation of undulator sources.

The authors thank the BESSY staff for their assistance. The financial support of the Bundesministerium für Forschung und Technologie and the European Economic Community is gratefully appreciated. One of us (N.M.K.) acknowledges the financial support of the Deutsche Forschungs Gemeinschaft.

[1] C. H. Heckenkamp, F. Schäfers, G. Schönhense, and U. Heinzmann, Z. Phys. D 2, 257 (1986).

[2] U. Heinzmann, J. Phys. B 13, 4353 (1980).

[3] A. Svensson, M. Müller, N. Böwering, U. Heinzmann, V. Radojevic, and W. Wijesundera, J. Phys. B 21, L179 (1988).

[4] A. Hausmann, B. Kämmerling, H. Kossmann, and V. Schmidt, Phys. Rev. Lett. 61, 2669 (1988).
[5] S. Southworth, U. Becker, C. M. Truesdale, P. H. Kobrin, D. W. Lindle, S. Owaki, and D. A. Shirley, Phys. Rev. A 28, 261 (1983).

[6] J. Jimémenez-Mier, C. D. Caldwell, and D. L. Ederer, Phys. Rev. Lett. 57, 2260 (1986).

[7] W. Kronast, R. Huster, and W. Mehlhorn, Z. Phys. D 2 , 285 (1986).

[8] M. Meyer, B. Müller, A. Nunnemann, Th. Prescher, E. v. Raven, M. Richter, M. Schmidt, B. Sonntag, and P. Zimmermann, Phys. Rev. Lett. 59, 2963 (1987).

[9] C. Kerling, N. Böwering, and U. Heinzmann, J. Phys. B 23, L629 (1990).

[10] T. J. Mcllrath and T. B. Lucatorto, Phys. Rev. Lett. 38, 1390 (1977).

[11] E. G. Berezhko and N. M. Kabachnik, J. Phys. B 12, 2467 (1977)

[12] C. H. Greene and R. N. Zare, Ann. Rev. Phys. Chem. 33, 119 (1982)

[13] K. Jost, J. Phys. E 12, 1006 (1979).

[14] K. Blum, Density Matrix Theory and Applications (Plenum, New York, 1981).

[15] S. Wakid, A. K. Bhatia, and A. Temkin, Phys. Rev. A 21 , $496(1980)$

[16] K. T. Chung, Phys. Rev. A 24, 1350 (1981).

[17] A. K. Bhatia, Phys. Rev. A 18, 2523 (1978).

[18] M. Rødbro, R. Bruch, and P. Bisgaard, J. Phys. B 12, 2413 (1979).

[19] S. Mannervik and H. Cederquist, Phys. Scr. 31, 79 (1985).

[20] D. Rassi, V. Pejcev, and K. J. Ross, J. Phys. B 17, 3535 (1977).

[21] M. Simsek, S. Simsek, and S. Erhoc, Chem. Phys. Lett. 91, 456 (1982). 